\title{
Does the A-line ARX-Index provide a reasonable assessment of anaesthetic depth in dogs undergoing routine surgery?
}

\author{
K E Joubert ${ }^{2}$
}

\begin{abstract}
The monitoring of anaesthetic depth is usually based on the subjective assessment of the patient. An objective assessment of anaesthesia has only recently become possible. The auditory-evoked response has predictable changes in response to increasing doses of anaesthetic agents. Recent advances have brought about a regression model with exogenous input of the auditory-evoked response, the A-line ARX-Index (AAI Index). The AAI Index is a dimensionless number between 0 and 100. This technology has been incorporated into the AEP (auditory-evoked potential) monitor that is utilised to assess anaesthetic depth in humans. This study was undertaken to determine if the AEP monitor was useful in dogs. Ten dogs were enrolled in the study. After a full clinical and otoscopic examination, dogs were premedicated with acetylpromazine and morphine. Anaesthesia was induced with thiopentone and maintained with halothane. End-tidal carbon dioxide, temperature, pulse oximetry, blood pressure and the electrocardiogram were monitored and recorded every 5 minutes. Anaesthetic depth was assessed as either being adequate or inadequate by the anaesthetist during surgery. An AEP monitor was attached to the patient and automatically collected AAI Index data. The anaesthetist was blinded to the AEP monitor. Following the completion of the surgical procedure, the patient was allowed to wake up with the AEP monitor attached. The AAI Index was analysed to compare adequate with inadequate anaesthesia during the period of surgery and awake with sleep data during recovery. All AAI Index values associated with inadequate anaesthesia were greater than 31 while adequate values were less than 35 . The difference between the groups was statistically significant and the power was 0.97 . Statistically, the awake and sleep values were significantly different with a power of 0.99 . From this study it can be concluded that the AAI Index shows good prospect for the evaluation of anaesthetic depth in dogs undergoing surgery. A larger study is needed to confirm these results.
\end{abstract}

Key words: AEP monitor, A-line ARX-Index, anaesthetic depth, auditory-evoked potential, canine.

Joubert K E Does the A-line ARX-Index provide a reasonable assessment of anaesthetic depth in dogs undergoing routine surgery? Journal of the South African Veterinary Association (2004) 75(3): 110-115 (En.). Anaesthesiology: Department of Companion Animal Clinical Studies, Faculty of Veterinary Science, University of Pretoria, Private Bag X04, Onderstepoort, 0110 South Africa.

\section{INTRODUCTION}

The evaluation of anaesthetic depth in anaesthesia commenced with the landmark paper of John Snow in 1847 describing the planes of anaesthesia ${ }^{8}$. Traditionally, awareness during anaesthesia has been based on sympathetic, haemodynamic, respiratory, ocular (mydriasis, myosis, 'fish eye') and neurological reflex (palpebral, pedal and corneal reflex) responses. Objective criteria for the assessment of anaesthetic depth are lacking ${ }^{5}$. Anaesthetic agent requirements vary between patients dependent on age, gender, cardiac output, clinical condition, serum proteins, preanaesthetic drug

${ }^{a}$ Anaesthesiology: Department of Companion Anima Clinical Studies, Faculty of Veterinary Science University of Pretoria, Private Bag X04, Onderstepoort, 0110 South Africa. E-mail: kenneth.joubert@up.ac.za Received: January 2004. Accepted: June 2004 administered and mass ${ }^{15}$. As a result, it is necessary to individualise the administration of anaesthetic agents according to the unique characteristics of each patient. Awareness under anaesthesia occurs in spite of the monitoring of anaesthetic $\mathrm{depth}^{8}$. An objective, reliable, repeatable, dose-dependent anaesthetic depth monitor, reflecting the state of arousal induced by surgical stimuli and the depression induced by anaesthetic agents, that is consistent between anaesthetic techniques is needed.

A monitor of anaesthetic depth must show graded changes in response to different concentrations of anaesthetic agents, indicate changes in response to surgical interventions and indicate light planes of anaesthesia and awareness ${ }^{34}$. The criteria for a reliable index of anaes- thesia is as follows: they should be valid for $95 \%$ of patients independent of surgery and anaesthesia, have a good distinction between awake and sleep states and must be able to detect states of awareness ${ }^{7}$.

Electroencephalography (EEG) has been used to determine anaesthetic depth through the determination of a number of derived parameters from the raw EEG. The bispectral index, the median and spectral edge frequencies have been used to assess anaesthetic depth and the effect of surgical stimulation on patients ${ }^{11,36}$. Spectral edge, median power, power bands and bispectral index have not proven to be repeatable and reliable between patients and different anaesthetic agents ${ }^{8}$. The bispectral index has been evaluated in the horse under isoflurane anaesthesia and has been found to be an unreliable indicator of anaesthetic depth ${ }^{13}$. The bispectral index was unable to differentiate between sedation and anaesthesia in horses ${ }^{13}$.

Anaesthesia depresses the ability of the central nervous system to respond to stimuli. Hearing is the last sense to disappear under anaesthesia and is gradually lost ${ }^{16}$. Auditory stimuli have been used to assess anaesthetic depth ${ }^{16,31}$.

The auditory-evoked response has been found to have a predictable and consistent dose-dependent response with various anaesthetic agents $s^{5,14,26,30,37}$. Volatile anaesthetic agents have been shown to exert similar effects on the auditory-evoked response based on comparison of minimum alveolar concentration multiples in humans ${ }^{14}$. The auditory-evoked response has also been used to show that neuromuscular blocking agents do not decrease a patient's requirement for anaesthesia in humans ${ }^{5,29}$.

The auditory-evoked response consists of a set of electrical impulses (brain waves) that are formed when a sound is delivered at the external auditory meatus. The electrical impulses generated travel from the cochlea through the brainstem and mid-brain to the cognitive centres of the brain. The first 7 waves represent the responses of the brain stem (brain stem auditory-evoked response, BAER) ${ }^{24,34,39}$. 
Table 1: The signalment of patients enrolled.

\begin{tabular}{|c|c|c|c|c|c|}
\hline Breed & $\begin{array}{c}\text { Age } \\
\text { (years) }\end{array}$ & $\begin{array}{l}\text { Mass } \\
(\mathrm{kg})\end{array}$ & Sex & Procedure & $\begin{array}{l}\text { Duration } \\
\text { (minutes) }\end{array}$ \\
\hline Cross-breed & 1 & 23 & Male & Tibia repair & 130 \\
\hline Labrador & 0.5 & 26 & Female & $\mathrm{OVH}$ & 140 \\
\hline Bull terrier & 2 & 24 & Male & Tibia repair & 100 \\
\hline Cross-breed & 5 & 29 & Male & Perineal hernia & 105 \\
\hline Fox terrier & 8 & 6.5 & Male & Tail amputation & 30 \\
\hline Spaniel & 6 & 18.5 & Female & Cutaneous tumour removal & 10 \\
\hline Boerboel & 4 & 35.2 & Male & Cruciate ligament repair & 95 \\
\hline German shepherd & 9 & 37 & Female & Biopsy & 20 \\
\hline Miniature pincher & 4 & 4.5 & Female & Caesarean & 75 \\
\hline Boerboel & 1 & 35 & Male & Castration & 30 \\
\hline Mean & 4.05 & 23.87 & & & 73.5 \\
\hline SD & 2.99 & 11.36 & & & 47.67 \\
\hline
\end{tabular}

As the electrical impulses move from the brain stem to the cortical structures, an early and late cortical response can be seen $^{34}$. The early cortical response occurs within $10-80 \mathrm{~ms}$ of the stimulus and is referred to as the middle latency component (MAER). Anaesthesia has been shown to have a reliable effect of altering the brain waves of the middle latency component ${ }^{28,34,39}$. The late cortical response occurs more than $80 \mathrm{~ms}$ after the stimulus and is a result of frontal cortex processing of the signal ${ }^{34}$. The auditoryevoked potential can be analysed by measurement of the amplitude and latencies of waves recorded 10-100 ms after auditory stimulation ${ }^{3}$. A regression model with exogenous input (ARX model) is used to analyse these amplitudes and latencies to derive a dimensionless number between 0 and 100 known as the A-line ARX-Index (AAI Index) ${ }^{16}$.

The auditory-evoked potential has been used for the assessment of anaesthetic depth in the rat ${ }^{1,2,18}$ and the pig ${ }^{22}$. Initial experimental assessment of auditoryevoked potential for the assessment of anaesthetic depth in dogs, indicated that it was useful ${ }^{25,28}$, but no studies on the clinical use of the AAI Index in dogs could be found. This study was undertaken to determine if the AAI Index has the ability to differentiate between adequate and inadequate planes of anaesthesia during surgery and between awake and sleep states during recovery in dogs.

\section{MATERIALS AND METHODS}

The Animal Use and Care Committee and the Research Committee of the Faculty of Veterinary Science approved this protocol. Ten dogs admitted to the Section of Small Animal Surgery for either hind limb orthopaedic or soft tissue surgery caudal to the thoraco-lumbar junction were enrolled into the study after informed consent from the owners had been obtained. The signalment of patients enrolled into the study is given in
Table 1. A full clinical examination, haematology, urine analysis and faecal examination was performed to ensure that no major clinical abnormalities were present before inclusion in the study. An otoscope was used to examine the external ear canal and the eardrum. The ear canal was cleaned to remove any waxy build up and the eardrum was examined to ensure that it was intact. A brief neurological examination was carried out to ensure that the dog could hear sounds and determine sound direction.

All dogs were premedicated with acetylpromazine (ACP, Centaur Laboratories, Isando) $(0.01 \mathrm{mg} / \mathrm{kg})$ and morphine (Micro Morphine, Micro HealthCare (Pty) Ltd, Bethlehem) (0.3-0.5 mg/kg). Anaesthesia was induced with thiopentone (Intraval Sodium, Rhône-Poulenc, Halfway House) $(10 \mathrm{mg} / \mathrm{kg}$ ) administered over 30 seconds. After the induction of anaesthesia, anaesthesia was maintained with halothane (Fluothane, AstraZeneca Pharmaceuticals, Sandton) titrated to the point where no response to surgical manipulation occurred. Epidural anaesthesia was provided to animals undergoing orthopaedic procedures. Ropivacaine (Naropin, AstraZeneca Pharmaceuticals, Sandton) $(1 \mathrm{mg} / \mathrm{kg})$ and morphine $(0.1$ $\mathrm{mg} / \mathrm{kg}$ ) was used.

Capnography (Capnomac Ultima, Datex-Ohmeda, Helsinki) was used to ensure that ventilation remained adequate with an end-tidal carbon dioxide concentration remaining between 4.5$5.5 \mathrm{KPa}$. An increase in partial pressure of carbon dioxide has been shown to influence the auditory-evoked response ${ }^{36}$. Respiratory rate was determined from the capnograph. Non-invasive blood pressure, electrocardiogram, temperature and pulse oximetry (Life Scope P, Nihon Kohden, Tokyo) were monitored continuously during the surgery. Body temperature was maintained within $1.0^{\circ} \mathrm{C}$ of normal $\left(38.5^{\circ} \mathrm{C}\right)$ by the application of a warm-water circulating blanket
(Norm-O-Temp, Cincinnati Sub Zero, Cincinnati) and/or warm air convection heater (Bair Hugger, Augustine Medical Inc, Eden Prairie). A decrease in body temperature has been shown to increase the latency and reduce the amplitude of waves $^{24,33,34,36}$.

Electrodes for the recording of the auditory-evoked potential were positioned as follows: the reference electrode was placed $1 \mathrm{~cm}$ rostral to the auditory meatus, the ground electrode on the tragus (lateral-caudal cartilage of the ear forming part of the lateral wall of the vertical ear canal) or $1 \mathrm{~cm}$ rostral to the auditory meatus of the opposite ear and an active recording electrode on the vertex (midline between the ears over the crista sagittalis externa) ${ }^{24}$. Earphones (Monitor Earphones, Danmeter) were placed into the external auditory meatus and held in place with tape.

The A-line ARX-Index (AAI Index) (AEP Monitor, version 1.5, Danmeter A/S, Alaris Medical Systems, Cape Town) was recorded after delivering a click to both ears at $75 \mathrm{~dB}$ with a pulse duration of $0.5 \mathrm{~ms}$ and a frequency of $6 \mathrm{~Hz}^{24,36}$. Changes in sound intensity may affect the recording of an auditory-evoked response and hence the same intensity was used throughout the study ${ }^{33}$. The AEP monitor extracts the auditory-evoked potential through regression analysis with an exogenous input to determine a dimensionless number between 0 and 100. A number closer to 0 is associated with a state of sleep while a number closer to 100 is associated with a state of wakefulness. The AEP monitor automatically generates a $\log$ file containing all recorded data. From the log file, the time, the AAI Index value, the resistance through the leads and event markers were extracted. Resistance through the lead system remained less than $2 \mathrm{k} \Omega$ throughout the study. AEP monitoring was instituted in the theatre once all physiological monitors had been attached. The screen was covered to blind 
Table 2: Descriptive statistics of results. $\boldsymbol{n}$ is the number of sampling points obtained from the 10 dogs.

\begin{tabular}{lrrrrrrr}
\hline & $\boldsymbol{n}$ & Mean & SD & Median & SE & Max & Min \\
\hline Adequate & 118 & 20.78 & 6.64 & 20 & 0.61 & 35 & 31 \\
Inadequate & 14 & 53.86 & 17.03 & 50 & 4.55 & 77 & 80 \\
Awake & 450 & 63.96 & 15.10 & 70 & 0.71 & 31 \\
Sleep & 450 & 26.00 & 7.29 & 25 & 0.34 & 49 \\
\hline
\end{tabular}

the anaesthetist to the AEP monitor.

Anaesthetic depth was assessed as either being adequate or inadequate by the anaesthetist. Anaesthesia was considered adequate if no response to surgical manipulation was evident, the pedal withdrawal reflex was just observable or absent, respiratory pattern remained regular with a regular pulse, jaw tone was weak and blood pressure was stable. The most defining criterion was a response to surgical stimuli. Anaesthesia was considered inadequate if a response to surgical stimuli was evident (movement, change in respiratory pattern, heart rate and blood pressure or an increase in muscle tone), if spontaneous movement occurred, reflexes returned or lacrimation was evident. The period for the collection of adequate/inadequate data started once all the monitoring devices had been attached and was stopped when the last skin suture had been placed.

Following the completion of the surgical procedure, the patient was allowed to wake up with AEP monitor attached. The patient was extubated when the swallowing reflex returned and this point was marked as an event on the AEP monitoring. AEP monitoring continued for as long as the patient would allow, the electrodes were displaced or the electromyographically generated signal interfered with the ability of the AEP monitor to determine an AAI Index. Two blocks of AAI Index data were then extracted from the log based on the point when extubation occurred. A point 1 minute before and after extubation was identified. Data were collected from a point 1 minute after extubation until the end of AEP monitoring and called awake data. An equal number of data points were collected in reverse, from the point 1 minute before extubation and called sleep data.

All physiological measurements were monitored continuously and recorded every 5 minutes. Final-year veterinary science and nurse students performed the anaesthesia during their practical rotation on anaesthesiology. The students were instructed to record all the physiology variables measured and to include a note at each time interval as to whether anaesthesia was adequate or inadequate.

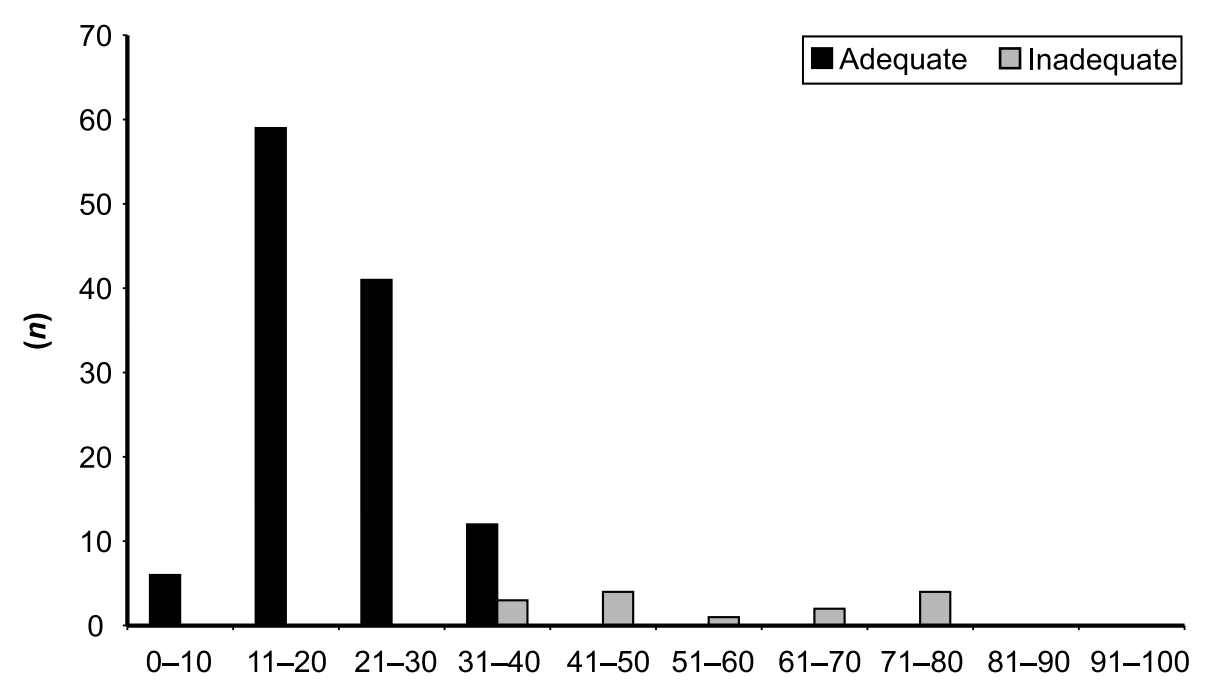

\section{AAI Index intervals}

Fig. 1: Bar graph of adequate and inadequate anaesthesia AAI values. The $y$-axis indicates the number of data points while the $x$-axis indicates data intervals.

AAI Index data were obtained from the automatically generated $\log$ in the AEP monitor. All data were collected manually on an anaesthetic monitoring form and transferred to a spreadsheet for analysis. Physiological data were not analysed, as there was no intention to determine any correlation between physiological data and the AAI index or anaesthetic depth. The AAI Index was analysed to compare adequate with inadequate anaesthesia during the period of surgery and awake with sleep data. The Mann-Whitney rank sum test was used to compare inadequate

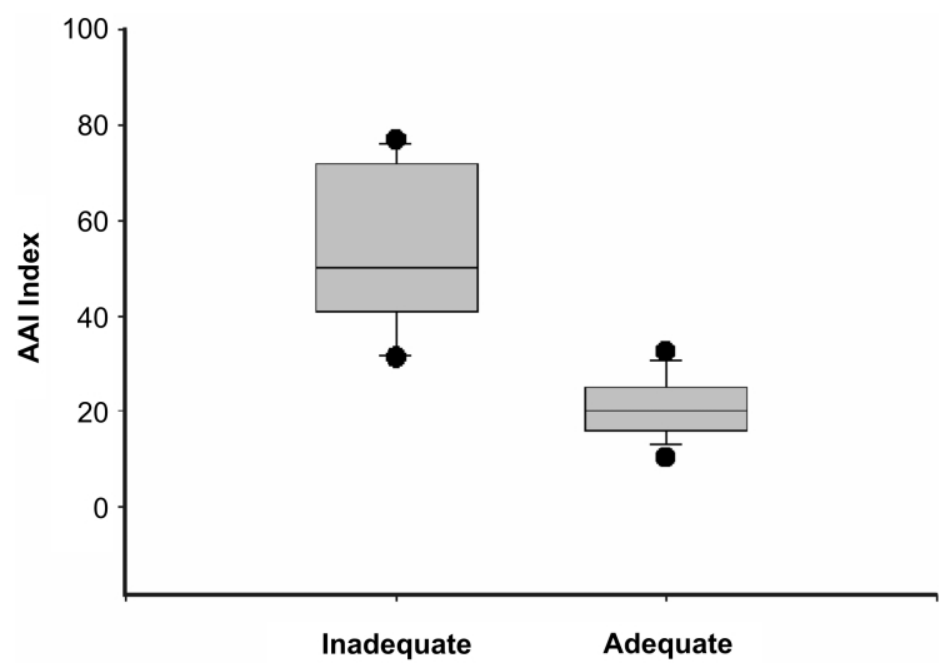

Fig. 2: A box plot of inadequate and adequate AAl values. The circles indicate outliers, the error bars the 5th and 95th percentiles and the line the median value. with adequate and awake with sleep data. Statistical significance was set at $P<0.05$. A power analysis was performed to determine the validity of the data collected. Sensitivity and specificity were determined.

\section{RESULTS}

One hundred and eighteen AAI Index values were collected for adequate anaesthesia and 14 values for inadequate anaesthesia during the surgical procedure (Table 2, Figs 1, 2). An area of overlap between 30 and 40 was evident with $21.4 \%$ 


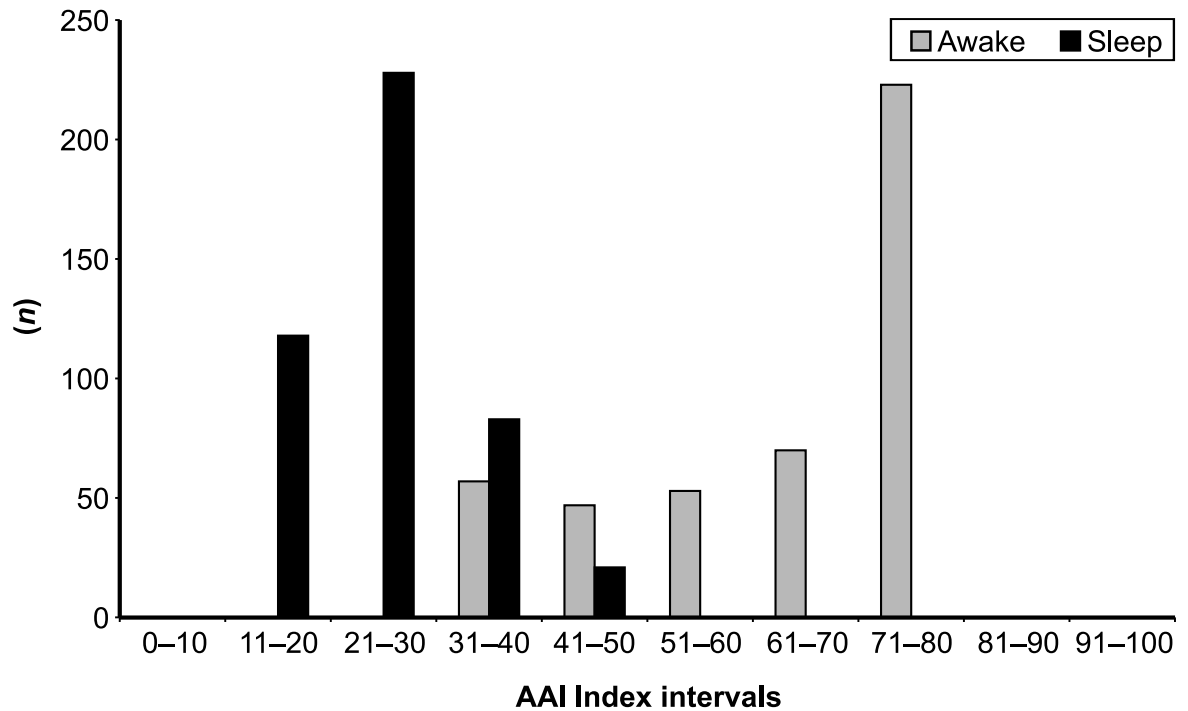

Fig. 3: Bar graph of awake and sleep AAl values. The $y$-axis indicates the number of data points while the $x$-axis indicates data intervals.

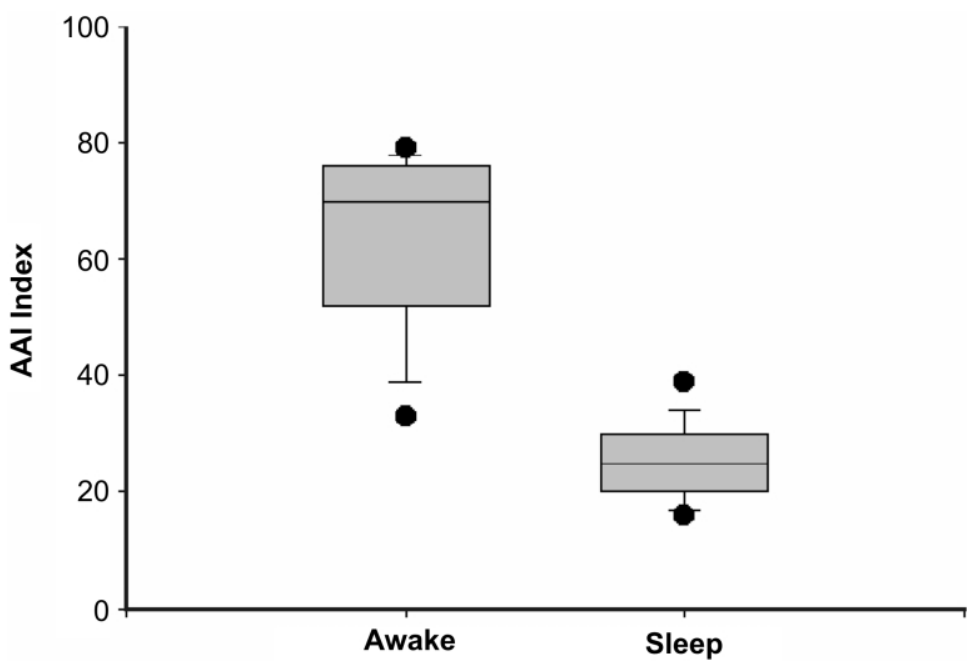

Fig. 4: Box plot of awake and sleep AAI values. The circles indicate the outliers, the error bars the 5th and 95th percentiles and the line the median value.

$(n=12)$ of inadequate values and $10.2 \%$ (3) of adequate values occurring in this area. Most periods of inadequate anaesthesia occurred just after the start of surgery. All AAI Index values associated with inadequate anaesthesia were greater than 31 while adequate values were less than 35. The difference between the groups was statistically significant $(P<$ $0.001)$. The power of this part of the study was 0.97 .

In the 2nd part of the study 450 sample points were collected from the point 1 minute after extubation and a similar number were collected 1 minute before extubation (Table 2, Figs 3,4). The area of overlap was greater in this part of the study and was between 31 and 50 . Twenty three per cent of awake and sleep data fell in this area. Statistically the 2 groups were significantly different $(P<0.001)$. During recovery it was found that AAI values increased after extubation but values would decrease again and some patients returned to a state similar to that of anaesthesia. If stimulation was applied (legs moved, jaw opened or a loud noise was made) the AAI values increased rapidly. A power of 0.99 was established.

When a cut-off value of 35 was taken for AAI Index (adequate/inadequate anaesthesia data), the results were $100 \%$ specific and $97.5 \%$ sensitive for adequacy of anaesthesia. At an AAI Index cut-off value of 30 , specificity decreased to $53.8 \%$ while sensitivity increased to $100 \%$. The specificity for the awake/sleep data for state of sleep at an AAI Index cut-off of 35, specificity was $93.6 \%$ and sensitivity was $92.1 \%$. If the cut-off was moved to 30 , the specificity for sleep moved to $81.7 \%$ but the sensitivity was $100 \%$.

\section{DISCUSSION}

This study showed that the AAI Index has the ability to indicate the adequacy of anaesthesia in dogs.

The auditory-evoked response can be influenced by a number of factors. The cardiac electrical activity ${ }^{27}$, arterial partial pressure of carbon dioxide ${ }^{36}$, body temperature $^{34}$, and the status of the external ear canal ${ }^{9}$. Cardiac electrical activity is a problem in children less than 2 years age $^{27}$. The changes have been ascribed to the high heart rate and the ratio of head size to body size, which normalises after the age of $2^{27}$. The effect of body mass on the AAI Index is unknown in dogs but the physiological basis may equally apply in veterinary medicine. Further evaluation of the effect of body mass and cardiac electrical activity on AAI values is required due to small size and high heart rates of a number of our patients. The arterial partial pressure of carbon dioxide and body temperature were monitored throughout the anaesthetic period. Hypothermia is a common complication under anaesthesia ${ }^{4,10,21}$. As the auditoryevoked potential is affected by hypothermia, the AEP monitor may be unreliable in hypothermic patients. These variables were controlled during this study to ensure that it remained in ranges shown to have a minimal effect on the auditoryevoked potential.

The ear canal and external ear drum were examined to ensure that they were intact. Conductive hearing loss has been described as result of otitis externa, rupture of the ear drum and blockage of the ear canal ${ }^{9,32}$. These factors should be borne in mind when auditory-evoked potential monitoring is considered for the assessment of anaesthetic depth. Bone conduction (vibrations) has been used to assess the auditory-evoked potential in patients with external ear disease $e^{23}$.

Anaesthetic agents have a dose-dependent effect on the auditory-evoked potential $^{7,16-18,20}$, and consideration as to the choice of premedications may be important. Morphine has been shown to have minimal effects on the auditoryevoked response $\mathrm{e}^{34}$ and has been used in several studies of the auditory-evoked response for the assessment of anaesthetic depth in humans and animals ${ }^{14,26,27,35-38}$. The effect of acetylpromazine on the AAI Index has been shown to be minimal ${ }^{28}$. The premedication protocol represented a combination drugs commonly used in South Africa ${ }^{19}$ and has minimal effects on the auditory-evoked potential. Epidural analgesia was determined by the procedure performed and utilised to provide the best analgesia for the patient. In humans, AAI Index values are correlated to the degree of sedation induced by midazolam and propofol in patients who have received epidural anaesthesia ${ }^{12}$. It is 
though that epidural anaesthesia has a minimal effect on the AAI Index. The effect of premedication and analgesic technique should not have had a major influenced the AAI Index in this study.

In rats, the AAI index is well correlated with anaesthetic depth and the potential for movement under anaesthesia ${ }^{18}$. An anaesthetic depth index of less than 35 was associated with a lack of response to deep pain ${ }^{18}$. An AAI index of less than 30 is associated with adequate anaesthesia in humans ${ }^{3}$. This correlates well with the AAI Index values recorded in our adequately anaesthetised patients. The AAI Index has the potential to detect the balance between stimulation and depression during surgery ${ }^{38}$. The arousal of several patients at the start of surgery is the result of a period of minimal stimulation during preparation and draping of the patient before surgery. The AEP monitor was able to identify this arousal by an increase in the AAI Index.

An AAI Index value of 35 or above allowed us to identify all patients who were inadequately anaesthetised in this study. An AAI Index value of 30 or less resulted in a number of patients who were adequately anaesthetised being declared inadequately anaesthetised. At an AAI Index of 35 or less, approximately $2.5 \%$ of patients may be inadequately anaesthetised according to our study. The criteria established for a good anaesthetic monitor indicates that it should be effective in $95 \%$ of our patients ${ }^{7}$. An AAI Index value of less than 35 does achieve this goal. Relatively few values were recorded for inadequately anaesthetised dogs and these results should be interpreted cautiously until more work is done to validate this. The larger awake/sleep data support the concept that an animal with an AAI Index of less than 30 is adequately anaesthetised. No surgical stimuli were applied during the collection of awake/ sleep data and a comparison between stimulated and non-stimulated patients may not be fair. If surgical stimulation was applied, higher values would have been recorded during the awake data period. The awake data were also influenced by myoelectrical activity. The AEP monitor automatically monitors myoelectrical activity. A high degree of muscle tone will affect the ability of the AEP monitor to analyse the auditory-evoked potential. When the signal (auditory-evoked response) and noise (for example: muscle activity) ratio is high, the monitor indicates that AAI Index is unreliable. The AAI Index may become unreliable during periods of shivering or other increases in muscle tone. The awake/sleep data cannot be used to assess the adequacy of anaesthesia for surgery.

Two important components of balanced anaesthesia are unconsciousness (sleep) and analgesia. The auditory-evoked response does not assess analgesia as such but rather the sleep component of anaesthesia ${ }^{6,29}$. During the collection of awake/ sleep data, dogs would wake from anaesthesia with extubation and then go back to sleep. With the onset of sleep, the AAI values decreased towards values obtained during anaesthesia but when the animal was disturbed, AAI values rapidly increased. Stimulation of the patient may be necessary to provide a more reliable AAI Index value associated with adequate anaesthesia for surgery.

From this study it can be concluded that the AAI Index shows promise for the evaluation of anaesthetic depth in dogs undergoing surgery. A larger study is needed to confirm the AAI Index value for adequate anaesthesia in dogs. An AAI Index of less than 30 should be considered in patients where movement maybe problematic during surgery. As the monitor indicates more a state of sleep than that of anaesthesia as such, patients with minimal stimulus may have a low AAI Index value and then respond suddenly when surgery is started. Stimulation of the patient during these quiet periods may help to produce more reliable results.

\section{ACKNOWLEDGEMENTS}

This study would not have been possible without the support of Alaris Medical Systems, South Africa, and in particular Vanessa Christensen and Lynette Olivier. Alaris Medical Systems generously supplied the equipment for this study. Quentin Bellingham, Alaris Medical Systems, is thanked for his technical input and the setting up the data transfer from the AEP monitor to a laptop computer. The surgeons, theatre and clinic staff and students at the Onderstepoort Academic Veterinary Hospital are thanked for their assistance and help.

\section{REFERENCES}

1. Antunes L M, Roughan J V, Flecknell P A 2001 Evaluation of auditory evoked potentials to predict depth of anaesthesia during fentanyl/fluanisone-midazolam anaesthesia in rats. Veterinary Anaesthesia and Analgesia 28: 196-203

2. Antunes L, Golledge H D R, Roughan J V, Flecknell P A 2003 Comparison of electroencephalogram activity and auditory evoked response during isoflurane and halothane anaesthesia in the rat. Veterinary Anaesthesia and Analgesia 30: 15-23

3. Barr G, Anderson R, Jakobsson J 2002 The effects of nitrous oxide on the auditory evoked potential index during sevoflurane anaesthesia. Anaesthesia 57: 736-739

4. Beal M W, Brown D C, Shofer F S 2000 The effects of perioperative hypothermia and the duration of anesthesia on postoperative wound infection rate in clean wounds: a retrospective study. Veterinary Surgery 29: 123-127

5. Brunner M D, Nathwani D, Rich P A Thornton C, Doré CJ, Newton D E F 1996 Effect of suxamethonium on the auditory evoked response in humans. British Journal of Anaesthesia 76: 31-37

6. Brunner M D, Nel M R, Fernandes R Thornton C, Newton D E F 2002 Auditory evoked response during propofol anaesthesia after pre-induction with midazolam. British Journal of Anaesthesia 89(2): 325-327

7. Capitanio L, Jensen E W, Filligoi G C, Makovec B, Gagliardi M, Henneberg S W Lindholm P, Cerutti S 1997 On-line analysis of AEP and EEG for monitoring depth of anaesthesia. Methods of Information in Medicine 36: 311-314

8. Drummond J C 2000 Monitoring depth of anesthesia. Anesthesiology 93: 876-882

9. Eger C E, Lindsay P 1997 Effects of otitis on hearing in dogs characterised by brainstem auditory evoked response testing. Journal of Small Animal Practice 38: 380-386

10. Evans A T, Sawyer D C, Krahwinkel D J 1973 Effect of a warm-water blanket on development of hypothermia during small animal surgery. Journal of the American Veterinary Medical Association 163: 2: 147-148

11. Gajraj R, Doi M, Mantzaridis H, Kenny G N C 1999 Comparison of bispectral EEG analysis and auditory evoked potentials for monitoring depth of anaesthesia during propofol anaesthesia. British Journal of Anaesthesia 82: 5: 672-678

12. Ge S J, Zhuang X L, Wang Y T, Wang Z D, Li H T 2002 Changes in the rapidly extracted auditory evoked potentials index and the bispectral index during sedation induced by propofol or midazolam under epidural block. British Journal of Anaesthesia 89: 2. 260-264

13. Haga H A, Dolvik N I 2002 Evaluation of bispectral index as an indicator of degree of central nervous system depression in isoflurane-anaesthetized horses. American Journal of Veterinary Research 63: 3: 438442

14. Heneghan C P H, Thornton C, Navaratnarajah M, Jones J G 1987 Effect of isoflurane on the auditory evoked response in man. British Journal of Anaesthesia 59: 277-282

15. Jacobs J R, Reves J G 1993 Effect site equilibration is a determinant of induction dose requirements. Anesthesia and Analgesia 76: $1-6$

16. Jensen E W, Lindholm P, Henneberg S W 1996 Autoregressive modeling with exogenous input of middle-latency auditoryevoked potentials to measure rapid changes in depth of anesthesia. Methods of Information in Medicine 35: 256-260

17. Jensen EW, Nebot A, Caminal P, Henneberg S W 1999 Identification of causal relationships between haemodynamics variables, auditory evoked potentials and isoflurane by means of fuzzy logic. British Journal of Anaesthesia 82: 1: 25-32

18. Jensen E W, Nygaard M, Henneberg S W 1998 On-line analysis of middle latency auditory evoked potentials (MLAEP) for monitoring depth of anaesthesia in laboratory rats. Medical Engineering and Physics 20: 722-728

19. Joubert K E 2000 Routine veterinary anaesthetic management practices in South 
Africa. Journal of the South African Veterinary Association 71: 3: 166-172

20. Litvan H, Jensen E W, Galan J, Lund J, Rodriguez B E, Henneberg S W, Caminal P, Landeira J M V 2002 Comparison of conventional averaged and rapid averaged, autoregressive-based extracted auditory evoked potentials for monitoring the hypnotic level during propofol induction. Anesthesiology 97: 351-358

21. Machon R G, Raffe M R, Robinson E P 1999 Warming with a forced air warming blanket minimizes anesthetic-induced hypothermia in cats. Veterinary Surgery 28: 301-310

22. Martoft L, Jensen E W, Rodriguez BE, Jørgensen, P F, Forslid, A, Pedersen, H D 2001 Middle-latency auditory evoked potentials during induction of thiopentone anaesthesia in pigs. Laboratory Animals 35: 353-363

23. Munro K J, Paul B, Cox C L 1997 Normative auditory brainstem response data for bone conduction in the dog. Journal of Small Animal Practice 38: 353-356

24. Myers L J, Redding R W, Wilson S 1985 Reference values of the brainstem auditory evoked response of methoxyflurane anesthetized and unanesthetized dogs. Veterinary Research Communications 9: 289-294

25. Nayak A, Roy R J 1998 Anesthesia control using midlatency auditory evoked potentials. IEEE Transactions on Biomedical Engineering 45: 4: 409-421

26. Newton D E F, Thornton C, Creagh-Barry P, Doré C J 1989 Early cortical auditory evoked response in anaesthesia; comparison of the effects of nitrous oxide and isoflurane. British Journal of Anaesthesia 62: 61-65

27. O'Kelly S W O, Smith D C, Pilkington S N 1995 The auditory evoked potential and paediatric anaesthesia. British Journal of Anaesthesia 75: 428-430

28. Pypendop B, Poncelet L, Verstegen J 1999 Use of midlatency auditory-evoked potentials as indicator of unconsciousness in the dog: characterisation of the effects of acepromazine-thiopentone, medetomidine-thiopentone and medetomidinebutorphanol-midazolam combinations. Research in Veterinary Science 67: 35-39

29. Richmond C E, Matson A, Thornton C, Doré C J, Newton D E F 1996 Effect of neuromuscular block on depth of anaesthesia as measured by the auditory evoked response. British Journal of Anaesthesia 76: 446-448

30. Sharpe R M, Nathwani D, Pal S K, Brunner M D, Thornton C, Doré C J, Newton D E F 1997 Auditory evoked response, median frequency and 95\% spectral edge during anaesthesia with desflurane and nitrous oxide. British Journal of Anaesthesia 78: 282-285

31. Stanski D R 2002 Monitoring depth of anaesthesia In Miller R D, Cucchiara R F, Miller E D, Reves J G, Roizen M F, Savarese J J (eds) Anesthesia (5th edn). Churchill Livingstone, Philadelphia: 1087-1116

32. Steiss J E, Wright J C, Storss D P 1990 Alterations in the brain stem auditory evoked eresponse threshold and latency-intensity curve association with conductive hearing loss dogs. Progress in Veterinary Neurology 1: 205-211

33. Stockard J J, Stockard J E, Sharbrough F W 1978 Nonpathologic factors influencing brainstem auditory evoked potentials. American Journal of EEG Technology 18: 177-209

34. Thornton C 1991 Evoked potentials in anaesthesia. European Journal of Anesthesiology 8: 89-107

35. Thornton C, Barrowcliffe M P, Konieczko K Ventham P, Doré C J, Newton D E F, Jones J G 1989 The auditory evoked response an indicator of awareness. British Journal of Anaesthesia 63: 113-115

36. Thornton C, Heneghan C P H, James M F M, Jones J G 1984 Effects of halothane and enflurane with controlled ventilation on auditory evoked potentials. British Journal of Anaesthesia 56: 315-323

37. Thornton C, Heneghan C P H, Navaratnarajah M, Jones J G 1986 Selective effect of althesin on the auditory evoked response in man. British Journal of Anaesthesia 58: 422-427

38. Thornton C, Konieczko K, Jones J G, Jordan C, Doré C J, Heneghan C P H 1988 Effect of surgical stimulation on the auditory evoked response. British Journal of Anaesthesia 60: 372-378.

39. Thornton C 2001 Auditory evoked response. Alaris Medical Systems, Cape Town 